\title{
N-BODY SIMULATIONS OF GALAXY FORMATION
}

\author{
Carlos S. Frenk \\ Physics Department \\ University of Durham \\ England
}

\begin{abstract}
Modern N-body techniques allow the study of galaxy formation in the wider context of the formation of large-scale structure in the Universe. The results of such a study within the cold dark matter cosmogony are described. Dark galactic halos form at relatively recent epochs. Their properties and abundance are similar to those inferred for the halos of real galaxies. Massive halos tend to form preferentially in high density regions and as a result the galaxies that form within them are significantly more clustered than the underlying mass. This natural bias may be strong enough to reconcile the observed clustering of galaxies with the assumption that $\Omega=1$.
\end{abstract}

\section{INTRODUCTION}

Modern N-body methods are a very effective tool to model the genesis of galaxies. They offer the freedom to chose realistic initial conditions and are able to incorporate mathematically intractable non-linear processes. With the computing power available today and the most sophisticated techniques it is possible to resolve the inner few kiloparsecs of a forming galaxy and at the same time follow the changing environment out to several megaparsecs. Traditional N-body techniques are applicable only to dissipationless systems. They are thus relevant to the study of dark halos and may also be relevant to the study of elliptical galaxies and the bulges of spirals. A treatment of dissipative phenomena is essential to model the formation of disks but attempts to include such effects are still in their infancy (Negroponte and White 1983, Carlberg 1987).

At the most basic level, a successful theory of galaxy formation must account for at least the following observed properties:

(i) Abundance

The number density of galaxies of different luminosity $L$ is measured observationally (eg. Efstathiou, et. al. 1987). Using an empirical relation between luminosity and a characterisitic velocity $V_{c}$, (Faber and Jackson 1976, Tully and Fisher 1977), the distribution function of $V_{c}$ can be obtained. This is a dynamical quantity which measures the specific binding energy of the galaxy. A remarkable fact is that about $80 \%$ of the stars in the Universe appear to occur in systems within a rather narrow range of $V_{c}: \sim 140-300 \mathrm{~km} / \mathrm{s}$ for disks and $\sim 200-450 \mathrm{~km} / \mathrm{s}$ for ellipsoids (White 1985). 
(ii) Internal structure

The mass density in the outer parts of spirals is inferred from their rotation curves to fall off approximately as $r^{-2}$, much less rapidly than the exponential light distribution of the disks (van Albada et. al. 1985). Little is known about the mass distribution around ellipsoidal systems but their luminosity profiles fall off more steeply than the massive halos of spirals, approximately as $r^{-3}$.

(iii) Angular momentum

Disks have a larger specific angular momentum than ellipsoidal components (eg. Fall 1983). Faint ellipticals and the bulges of spirals have similar rotational properties; they rotate faster than bright ellipticals (Davies et. al. 1983).

(iv) Spatial distribution

To first order the clustering of bright galaxies on scales up to $\sim 10 h^{-1} M p c$ is described by the two-point correlation function $\xi(r) \sim\left(r / 5 h^{-1} M p c\right)^{-1.8}$ (Davis and Peebles 1983). There are, however, important differences in the distributions of different kinds of galaxies. Ellipticals tend to occur in denser environments and are correspondingly more clustered than spirals (Dressler 1980, Davis and Geller 1976) and there is an indication that the characteristic luminosity may be somewhat higher in clusters than in the field (Giovanelli, this volume).

(iv) Morphological types

These are summarized in the "Hubble sequence" of galaxy types.

Until recently most $\mathrm{N}$-body studies considered the formation of a single galaxy and focused primarily on point (ii). For example, van Albada (1982) showed that the collapse of an initially cool and clumpy distribution of collision-free stars would relax to a system with a density profile similar to that of elliptical galaxies. McGlynn (1984) found that a wide range of initial conditions could produce similar profiles. Calberg et. al. (1986), on the other hand, argued that some amount of energy dissipation is required to produce an elliptical galaxy profile from the recollapse of an initially expanding random distribution. While these and other related studies have been valuable in understanding the physics of gravitational collapse, they do not provide an explanation of how the assumed initial conditions would arise nor do they take into account the evolution of the cosmic environment. It is evident that the issues listed above cannot be addressed by considering the formation of a single galaxy in isolation. Rather, a full cosmological model which can simultaneously explain the existence of galaxies, clusters and other observed largescale structures is required. Such models have only recently been formulated and the study of the modes of galaxy formation which they imply is only now beginning.

The best studied and in many ways the most successful of the new cosmogonies is the cold dark matter (CDM) theory ( $c f$ Blumenthal et. al. 1984). In the remainder of this article I will restrict attention to this specific scenario. After a brief summary of its main assumptions and successes, I will discuss in some detail the formation history of galactic halos, their internal structure and abundance and the relation between the spatial distribution of halos, of the galaxies they harbour and the overall distribution of mass. This work is part of an on-going collaboration with Marc Davis, George Efstathiou and Simon White.

\section{THE COLD DARK MATTER COSMOGONY}

In its simplest form the CDM model assumes that the universe has the Einstein-deSitter geometry $(\Omega=1)$ and that its density is dominated by weakly interacting 
elementary particles which provide the missing mass in galaxies and clusters. In addition it assumes that density fluctuations at early times have the scale-invariant structure predicted to result from quantum fluctuations in an inflationary epoch (Peebles 1982, Bond and Szalay 1983). In order to reconcile the assumption of a flat geometry with observations of galaxy clusters, it is necessary for galaxy formation to favour the dense regions to which virial methods can be applied. It has been further assumed that in the CDM model galaxies form only near high peaks of the linear density field and as a result are biased relative to the mass. (Bardeen et. al. 1986, Davis et. al. 1985).

These assumptions lead to a fully specified model in which the mass distribution is determined by only 2 "free" parameters, the initial amplitude of the fluctuation spectrum and a linear scale fixed by the Hubble constant. In the high peak model, the galaxy distribution is specified by 2 further parameters, the abundance of galaxies and the strength of the bias in the galaxy distribution. All four parameters can be simultaneously fixed by matching the model predictions to the observed galaxy correlations. There is then no further freedom in the model and it can be tested against observations on larger and smaller scales.

The evolution of a CDM universe up to the present day can be calculated by numerical simulation. Structure is found to grow by hierarchical clustering. Objects aggregate very rapidly from subgalactic to galactic scales; thereafter growth continues more slowly. The behaviour of a CDM universe on scales ranging from those of galactic halos to those of superclusters of galaxies has been studied by my collaborators and I in a series of recent papers. (Efstathiou et. al. 1985, Davis et. al. 1985, Frenk et. al. 1985,1987, White et. al. 1987a,b; for reviews see Frenk 1986,1987 and White 1986). These and other related studies have shown that the CDM cosmogony accounts for:

- On intermediate scales ( 1-10Mpc)

(i) The galaxy two- and three-point correlation functions

(ii) The pairwise relative peculiar velocity of galaxies

(iii) The properties of galaxy groups (Nolthenius and White 1986)

(iv) The abundance, structure, luminosity function and mass-to-light ratio of Abell clusters

(v) Our infall velocity towards Virgo

(vi) The isotropy of the microwave background (Bond and Efstathiou 1984, Vittorio and Silk 1984).

- On large scales $(\sim 10-100 \mathrm{Mpc})$

(i) Voids as large as the one in Bootes (Kirshner et. al. 1987)

(ii) Filaments like the Perseus-Piscis chain (Haynes and Giovanelli 1986)

(iii) Bubbles similar to those found in the extended CfA survey (de Lapparent et. al. 1986)

(iv) The velocity of the Local Group relative to the microwave background

(v) The rms peculiar velocity of clusters measured by Aaronson et al (1986)

- On galactic scales

(i) Flat rotation curves in the outer parts of spiral galaxies

(ii) The abundance and potential well depths of galactic halos

Significant discrepancies with present data are found only on the largest scales where the model may not produce sufficient superclustering. In particular, the predicted amplitude of cluster-cluster position and velocity correlations is smaller than the values quoted by Bahcall and Soneira (1983). The large-scale streaming motions recently discussed by Collins et. al. (1986) and Dressler et. al. (1987) have 
been claimed to be inconsistent with CDM (Vittorio et al 1986). However, through a reanalysis of most of these data Kaiser (1987) has reached the opposite conclusion. Finally, the anisotropies in the microwave background on degree scales recently detected by Davies et. al. (1987) are larger than expected, but the amplitude of the primordial contribution is as yet unclear.

Although early on the introduction of the high peak model was a very useful and influential concept, the CDM model has now been developed to the stage where finding a physical explanation for why galaxies should be biased has become a central issue. Several mechanisms have been proposed that could inhibit galaxy formation in voids, but their nett effect on the galaxy distribution remains to be quantified ( $c f$ Rees, this volume). In this article I will argue that the required bias arises naturally from gravitational evolution in a CDM universe. Thus the CDM cosmogony is entirely specified by the distribution of mass; it is a 2 parameter model.

\section{THE FORMATION OF DARK HALOS}

To investigate the formation history of galactic halos in the CDM cosmogony, Davis Efstathiou, White and I recently carried out a series of high resolution N-body simulations. Prelimary reports have been published elsewhere (Frenk et. al. 1985, Frenk 1987) and more detailed accounts will appear in forthcoming papers (Frenk et. al. 1987, White et. al. 1987a). We followed the evolution of 9 cubic patches of a CDM universe of initial physical size $2 \mathrm{Mpc}$ from a redshift of 6 to the present day. (Here and below $H_{o}=50 \mathrm{~km} / \mathrm{s} / \mathrm{Mpc}$, the value required by our standard model.) Because we are interested in environmental effects, these 9 regions do not all have the same mean density. Instead they are divided into 3 ensembles of 3 calculations each. One ensemble has the critical density, and the other 2 differ from it by $\pm 9.4 \%$ initially, corresponding! to $1 \sigma$ upward and downward mass fluctuations on the scale of the initial box. These differences take into acount the modulating effect of wavelengths larger than the simulation volume in an approximate way. The simulations were carried out using the $\mathrm{P}^{3} \mathrm{M}$ technique (Efstathiou et. al. 1985). They have 32768 particles of mass $\sim 6 \times 10^{9} \mathrm{M}_{\odot}$ and a resolution of $\sim 2 \mathrm{kpc}$ at the start and $\sim 14 \mathrm{kpc}$ at the end of the simulations.

A qualitative impression of the formation paths of halos can be gained by plotting the projected positions of the particles that end up in the halos. A selection of objects chosen to span the range of masses and formation histories is shown in Figure 1. Between $z=2.5$ and $z=1$, evolution is dominated by mergers, often involving two or more lumps of roughly comparable size. $\mathrm{By} z=1$, most halos have developed a dominant central core. Many evolve to their final state in a fairly quiescent fashion, others accrete small satellites and a few undergo major merger events. At the final time all the halos appear smooth and centrally concentrated. The largest clump in the figure has a characteristic velocity of $403 \mathrm{~km} / \mathrm{s}$ and a rather violent recent history. This object is so massive that we will be required to interpret it as the common halo of a galaxy group rather than as the halo of a single galaxy. Objects like this are rare in the simulations and are not representative of the typical formation paths of galactic halos in the CDM cosmogony.

We can attempt to quantify the varied formation histories illustrated in the figure in two ways. Firstly let us consider the fate of halos that have formed at $z=2.5$ by asking how many of them survive at later times. At redshift $z$, we measure the mass of the largest subset of the original particles which remain as a bound object. If this mass has dropped below half of the original halo mass, the halo can be said to have been disrupted. This usually occurs when it has merged with a comparable or larger system. According to this definition, only about 20 of the halos present at $z=2.5$ remain intact by $z=0$. The disruption rate depends 
on the mass. Smaller halos are disrupted earlier than more massive ones. Secondly, let us consider the history of the progenitors of present day halos. At $z=2.5$, most had two or more precursors of similar mass and the largest of these contained only between $\sim 1 / 3$ and $\sim 1 / 30$ of the final mass. By $z=1$, about half of the progenitors have already acquired over half of the final mass and the second largest progenitor in each case has less that $\sim 1 / 10$ of it. These systems evolve to their final state by accretion of a moderate amount of material onto a dominant core. Finally, by $z=0.4$ most halos have acquired most of their final mass, and only about $1 / 3$ undergo major mergers thereafter.

\section{THE STRUCTURE OF DARK HALOS}

\section{- Rotation curves}

The potential wells of dark halos can be characterised by a "Rotation curve" calculated as $V(r)=[G M(r) / r]^{1 / 2}$, where $M(r)$ is the mass within a sphere of radius $r$ centred on the halo. For most halos the rotation curves turn out to be flat at radii larger than the resolution limit of the calculations (cf Fig. 1 of Frenk et. al. 1985 and Fig. 3 of Frenk 1987); they resemble the measured rotation curves in the outer parts of spiral galaxies (Rubin et. al. 1985). Only for the most massive halos do we find rotation curves that rise significantly at $50-100 \mathrm{kpc}$. These are the objects which have undergone most merging, and in some cases their rotation curves reach values well above those typical of spiral galaxies. Such objects must be identified with the halos of ellipticals or of small groups of galaxies. The generation of flat rotation curves seems to be closely related to the shape of the CDM fluctuation spectrum (Quinn et. al. 1986, Davis et. al. 1987).

\section{- Angular momentum}

Most halos tend to be slowly'rotating with typical values of $v / \sigma \sim 0.1$, where $v$ is the bulk rotation speed and $\sigma$ the $r m s$ velocity dispersion. The low rotation speeds are partly due to the outward transfer of angular momentum which occurs as substructure is erased; this purely gravitational process can be very efficient at spinning down the inner regions of dense clumps (Frenk et. al. 1985, Barnes and Efstathiou 1986).

- Shapes

Dark halos are generally triaxial with, perhaps, a slight preference for near prolate configurations. The axial ratios can be quite extreme: in a few systems the ratio of the two longest axes approaches 3 , and a ratio of 2 is quite common. There is a weak tendency for the centres of halos to be rounder than their outer regions. There is no correlation of shape with rotation speed. Halos are flattened by velocity anisotropies rather than by rotation.

\section{THE ABUNDANCE OF DARK HALOS}

The population of dark halos at any given time is established by a competition between creation and destruction processes. We describe each halo by a characteristic velocity $V_{c}$, defined as the value of its rotation curve at the boundary of a sphere within which the mean density is 1000 times the present critical density. The radius and mass of this sphere are related to $V_{c}$ by $(r / 180 \mathrm{kpc})=\left(V_{c} / 200 \mathrm{~km} / \mathrm{s}\right)$ and $\left(M / 1.65 \times 10^{12} \mathrm{Mo}\right)=\left(V_{c} / 200 \mathrm{~km} / \mathrm{s}\right)$. The abundance history is illustrated in Figure 2 where the cumulative distribution of $V_{c}$ is plotted as a function of redshift for the three ensembles of simulations. $N\left(V_{c}\right)$ is defined as the comoving number 
264
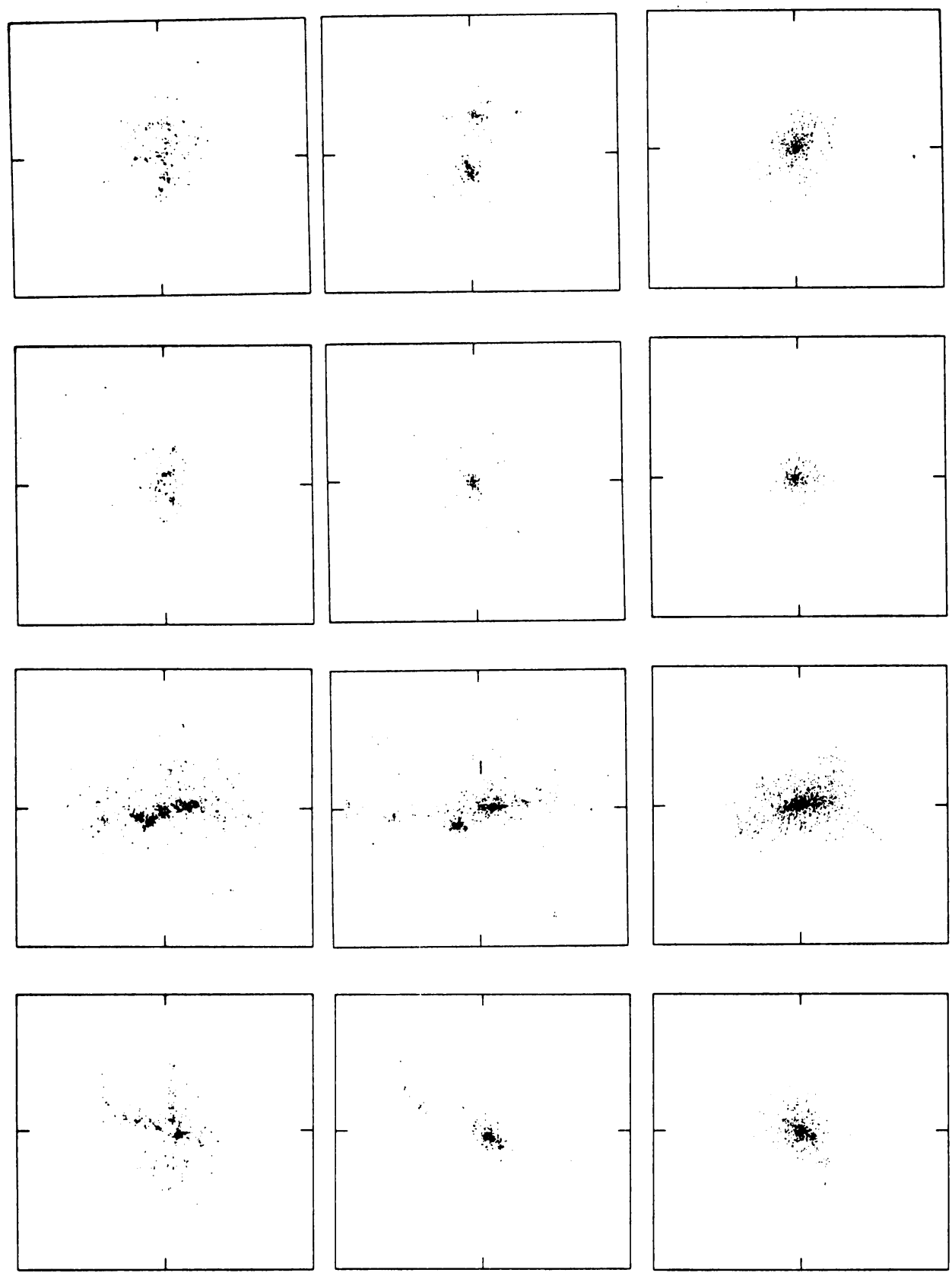

https://doi.org/10.1017/S0074180900136137 Published online by Cambridge University Press 

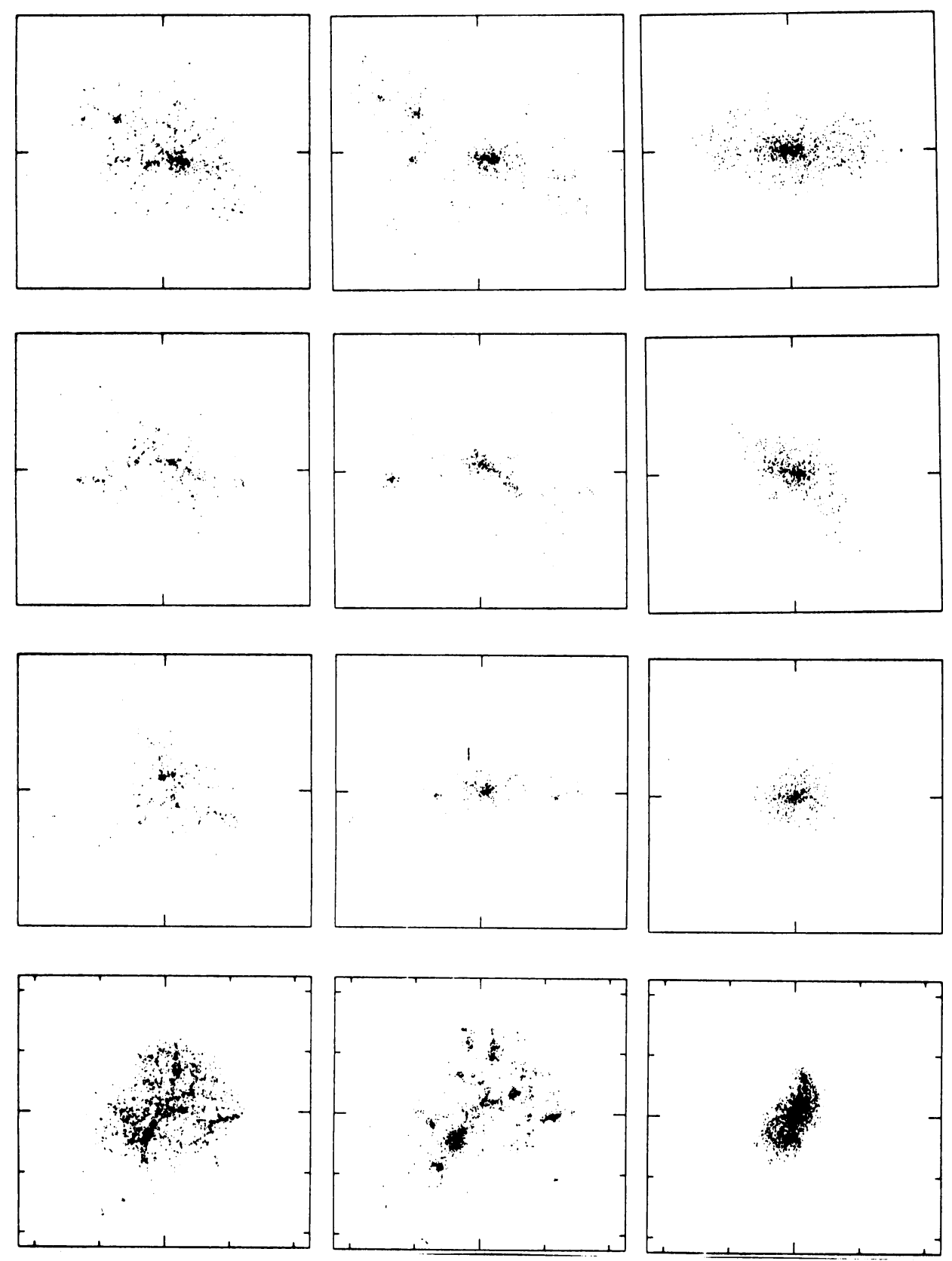

Figure 1. Time evolution of the particles which end up in selected halos. The three epochs shown correspond, from left to right, to $z=2.5,1$, and 0 . From top to bottom, the "circular velocities" of the halos at the present epoch are: 160, 180, 285, 219, 263, 204, 235 and $403 \mathrm{~km} / \mathrm{s}$. 
density of halos with circular velocity exceeding $V_{c}$. The following points are noteworthy:

(i) By $z=2.5$ halos with a wide range of values of $V_{c}$ have collapsed, but the bigger halos collapse only fairly recently.

(ii) The abundance of halos of any given $V_{c}$ increases at first and then levels off or, in the case of small halos, begins to decline as mergers become dominant.

(iii) There are marked differences in the three ensembles indicating that the abundance of halos is a strong function of environment. At any given time there are more halos of a given $V_{c}$ in the overdense than in the underdense simulations and the biggest halos form only in the former. This variation reflects the fact that fluctuations grow faster in a closed than in an open universe. It causes the statistical properties of the sites of galaxy formation to differ from those of the underlying mass distribution in the sense required by models of biased galaxy formation. This natural bias will be explored further in Section 6 .

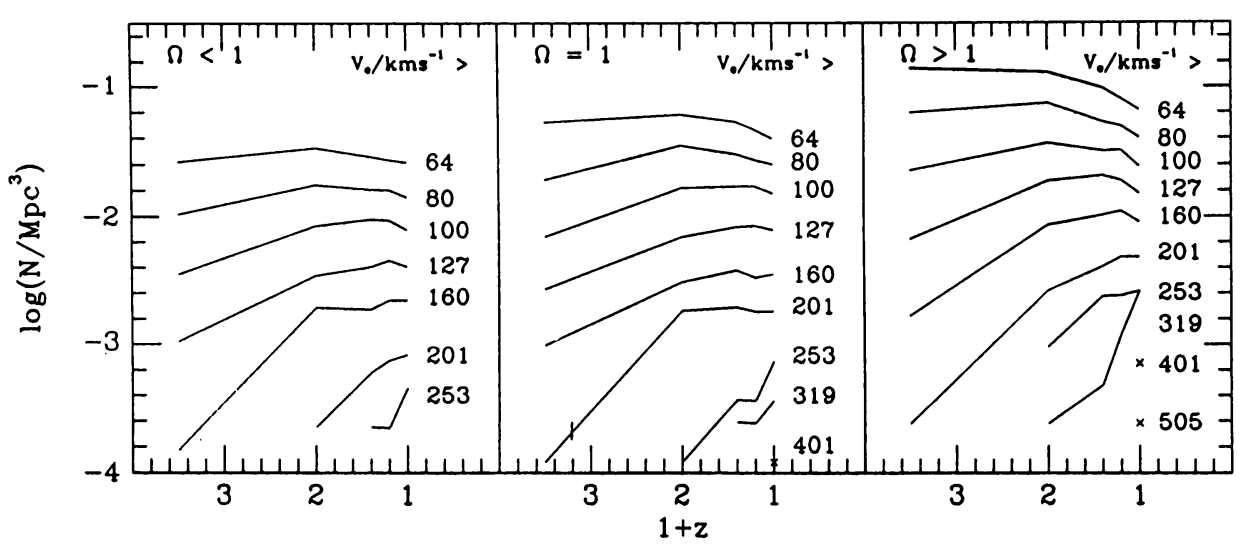

Figure 2. Time evolution of the cumulative distributions of characteristic velocities. The central panel corresponds to the ensemble with the critical density and the left and right panels to the underdense and overdense ensembles respectively. Each curve gives the number of halos per unit volume with characteristic velocity greater than the value shown.

To compare the predicted abundance of dark halos with the observed abundance of galaxies, we proceed as follows. We make the crude assumptions that every halo contains one and only one galaxy, and further, that the luminosity of the galaxy, $L$, is a monotonic function of $V_{c}$. With each halo of circular velocity $V_{c}$ we associate a value of $L$ by obtaining the luminosity for which the observed cumulative number density of galaxies equals the calculated cumulative number density of halos for that value of $V_{c}$. If, under our assumptions, the abundance of halos is correct a plot of $V_{c}$ versus $L$ for the halos should agree with the observed relation for galaxies. This comparison is shown in Figure 3. The filled squares give the model predictions and the other symbols correspond to observational data: the dashed line to the Tully-Fisher relation (Aaronson and Mould 1983) the dotted line to the Faber-Jackson relation (Davis et. al. 1983) and the dot to the study by Bothun et. al. (1985). The agreement between the CDM predictions and the data is remark- 
ably good. There are, however, several uncertainties in this comparison. Apart from uncertainties in the normalisation of the galaxy luminosity function and in the $L-V$ relations, our procedure for counting halos in the simulations is clearly an oversimplification. As we have seen the largest objects that form must correspond to the common halo of a group of galaxies. To correctly take this into account would require detailed modelling of galaxy formation which is beyond the scope of our present techniques. The agreement with the data does, however, show that the CDM model predicts about the correct abundance of galaxies. This is a remarkable success especially since there are no adjustable parameters in this prediction.

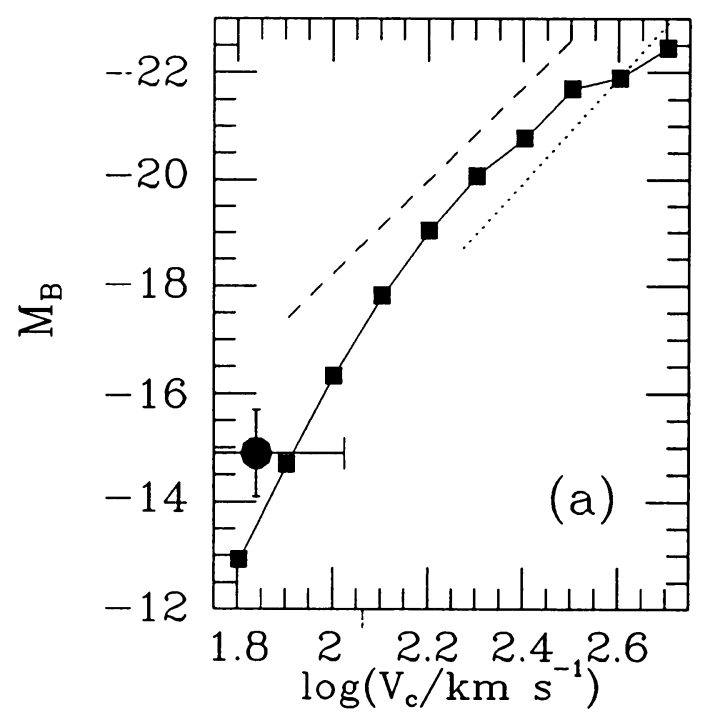

Figure 3. The "Tully-Fisher" relation for halos in the simulations. The " $B_{T}$ magnitude" of the "galaxy" associated with each halo is plotted as a filled square against characteristic velocity. The remaining symbols correspond to observational data described in the text.

\section{NATURAL BIAS}

To quantify the strength of the natural bias seen in the previous section, simulations of regions large enough to study the clustering of galaxies reliably are required. We ran 3 simulations of cubes of side $50 \mathrm{Mpc}$ using 262144 particles of mass $3.5 \times 10^{10} \mathrm{M}_{\odot}$ and a force resolution of $50 /(1+z) \mathrm{kpc}$. As before, there are no free parameters in specifying the distribution of mass, including that of dark halos, but the distribution of galaxies is uncertain. Our techniques do not allow us to study the behaviour of the dissipative gas from which galaxies must form. We are thus forced to put in galaxy formation and galaxy merging in a way which although plausible remains somewhat ad hoc. At various stages during the evolution of a model we identify the most strongly bound particle in each dark matter halo as the "galaxy" which had condensed within it. We then adopt simple algorithms to model galaxy mergers and to avoid multiple galaxy formation within the same 
halo. The results described below are not especially sensitive to these procedures which are described in detail elsewhere (White et. al. 1987a).

The spatial autocorrelation functions $\xi(r)$ for "galaxies" with $V_{c}>100 \mathrm{~km} / \mathrm{s}$ and $V_{c}>250 \mathrm{~km} / \mathrm{s}$ and for the mass distribution are shown in Figure 4. On all scales the galaxy autocorrelation functions have a higher amplitude than that of the mass and this amplitude increases with $V_{c}$. Over the separation range plotted, the mean correlation enhancement is about 1.8 for $V_{c}>100 \mathrm{~km} / \mathrm{s}$ and 5 for $V_{c}>250 \mathrm{~km} / \mathrm{s}$. The distribution of galaxies is thus significantly biased with respect to the mass. This natural bias arises because clumps in a high density region collapse earlier and accrete faster than similar objects in a low density region ( $c f$ Figure 3). As a result the typical mass and velocity dispersion of clumps is greater in protoclusters than in protovoids. The dark halos themselves turn out to be more strongly clustered than the mass. For "galaxies" with $V_{c}>250 \mathrm{~km} / \mathrm{s}$ the correlations are comparable to those of observed bright galaxies (represented in the figure by a dashed line of slope -1.8). Natural biasing thus appears to be strong enough to reconcile the dynamics of galaxy clustering with a flat universe. The model predicts that the strength of galaxy clustering should depend on luminosity. There is little evidence for this in present samples, but good statistics are available for only a narrow range of luminosities.

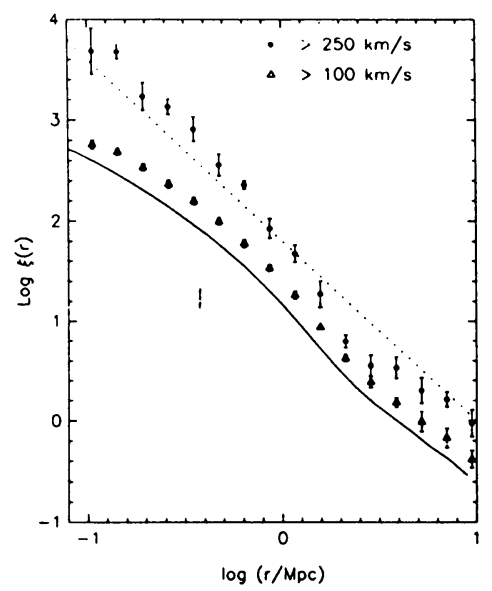

Figure 4. Spatial autocorrelation functions. The solid line shows the mass correlations, open triangles and filled circles show the correlations of "galaxies" with $V_{c}>100$ and $250 \mathrm{~km} / \mathrm{s}$ respectively. The dashed line shows the power-law $x i(r)=(r / 10 M p c)^{-1.8}$ which approximately describes the clustering of bright galaxies.

An important feature of natural biasing is that dwarf galaxies are not predicted to fill in the voids in the distribution of bright galaxies. The same structures are delineated by the mass, the faint and the bright galaxies, but the contrast increases with $V_{c}$. In regions devoid of bright galaxies, the mass density is also low and as a result these regions tend to be empty of fainter objects or at least to contain very few of them. It is therefore not surprising that searches for dwarfs in voids have been unsuccessful (Eder et. al. 1987). A direct test of natural biasing would require measuring the correlation function of galaxies of widely different intrinsic luminosities. 


\section{CONCLUSIONS}

I conclude by summarising what $\mathrm{N}$-body simulations tell us about the questions of when, how, and where do galaxies form in the CDM cosmogony.

\section{- When}

Galaxy formation is predicted to be a recent and protracted process which continues until the present day. Halos of galactic size halos are assembled in abundance only after $z \sim 3$. This does not preclude significant star formation in smaller units at somewhat earlier times.

\section{- How}

Most of the dark halos that form at early times, particularly the smaller ones, are later disrupted by mergers. Most present day halos had at least two progenitors of similar mass at $z>1$. Prior to this time galaxy formation was dominated by violent dynamical effects. The merging rate which is sensitive to the mean density of the galaxy environment, declines after $z \sim 1$. An extended period of quiescent evolution would seem to be required for high angular momentum gas to settle into centrifugal equilibrium and form a disk. The abundance of halos that are relatively undisturbed since $z \sim 1$ turns out to be similar to the observed abundance of spirals. It remains to be seen whether these halos are indeed sufficiently quiet and whether such recent formation of spiral disks is consistent with observations. Mergers of preexisiting stellar systems are likely to lead to the formation of bright elliptical galaxies (Toomre 1977, Fall 1979). Indeed, the outward transport of angular momentum by non-linear gravitational effects as substructure is erased causes the central regions of merger remnants to be slowly rotating. Galaxy merging, however, cannot be complete. If the CDM model is to be viable some galaxies must often survive the merger of their halos and assemble into virialized groups and clusters. A plausible but ad hoc treatment!of galaxy mergers suggests that this may well be the case.

\section{- Where}

Because the growth rate of gravitational fluctuations is density dependent, massive dark halos tend to form preferentially in high density regions. Their locations are closely related to the peaks of a suitably smoothed representation of the linear density field. As a result halos cluster more strongly than the mass, and this bias increases with the potential well depth of the halos considered. Its effect is enhanced by the fact that the more massive halos often contain several galaxies. A simple prescription for identifying galaxies within dark halos shows that this gravitational or natural bias may be strong enough to reconcile the dynamics of galaxy clustering with the theoretical imperative of a flat universe.

A great virtue of the CDM cosmogony is that is sufficiently well specified that many observable properties can be calculated reliably $a b$ initio. It is particularly encouraging that the same model which fits the clustering of galaxies and the luminosity function and masses of Abell clusters also reproduces the inferred abundance and structure of galactic halos. At the same time it offers a simple explanation for the geometry of our Universe.

Acknowldegements. I thank Marc Davis, George Efstathiou and Simon White for our enjoyable collaboration and for allowing me to discuss our results prior to joint publication. I am also grateful to Nick Kaiser and Martin Rees for many valuable discussions. This work was supported in part by a travel grant from NATO and by grants from the SERC and the Nuffield Foundation. 


\section{REFERENCES}

Aaronson, M., Bothun, G., Mould, J., Huchra, J.P., Schommer, R.A. and Cornell, M.E., 1986. Astrophys. J., 302,206.

Aaronson, M. and Mould, J., 1983. Astrophys. J., 265,1.

van Albada, T.S., Bahcall, J.N., Begeman, K. and Sanscisi, R., 1985. Astrophys. J., 295,305.

van Albada, T.S. 1982. Mon. Not. R. astr. Soc., 201,939.

Bahcall, N. and Soneira, R., 1983. Astrophys. J., 270,20.

Bardeen, J.M., Bond, J.R., Kaiser, N. and Szalay, A.S., 1986. Astrophys. J., 304,15 .

Barnes, J. and Efstathiou, G. 1986. Preprint.

Blumenthal, G.R., Faber, S.M., Primack, J.R. and Rees, M.J., 1984. Nature, 311,517 .

Bond, J.R. and Efstathiou, G. 1984. Astrophys. J., 285,L45.

Bond, J.R. and Szalay, A.S., 1983. Astrophys. J., 274,443.

Bothun, G.D., Mould,J.R.,Wirth,A. and Caldwell,N. 1985. Astron. J., 90, 697.

Carlberg, R.G., 1987. Preprint.

Carlberg, R.G., Lake, G. and Norman, C.A., 1986. Astrophys. J., 300,L1.

Collins, A., Joseph, R.D. and Robertson, N.A. 1986. Nature, 320, 506.

Davies, R.D. et. al. 1987. Nature, 326, 462.

Davies, R.L., Efstathiou, G., Fall, S.M., Illingworth, G. and Schechter, P.L., 1983. Astrophys. J., 266, 41.

Davis, M., Efstathiou, G., Frenk, C.S. and White, S.D.M., 1985. Astrophys. J., $292,371$.

Davis, M., Frenk, C.S., White, S.D.M. and Efstathiou, G., 1987. Preprint.

Davis M. and Geller, M.J., 19876. Astrophys. J., 208, 13.

Davis, M. and Peebles, P.J.E., 1983. Astrophys. J., 267, 465.

Dressler, A. 1980. Astrophyis. J.,1236, 351.

Dressler, A., Faber,S.M., Burstein, D., Davies, R.L., Lynden-Bell, D., Terlevich, R.J. and Wegner, G., 1987. Astrophys. J., 313, L37.

Eder, J., Schombert, J.M., Dekel, A. and Oemler, A., 1987. Preprint.

Efstathiou, G., Davis, M. Frenk, C.S. and White, S.D.M., 1985. Astrophys. J. Supp., 57, 241.

Efstathiou, G., Ellis, R.S. and Peterson, B.A., 1987. Preprint.

Faber, S.M. and Jackson, R.E., 1976. Astrophys. J., 204, 668.

Fall, S.M., 1979. Nature, 281, 200.

Fall, S.M., 1983. In Internal Kinematics and Dynamics of galaxies, IAU Symposium No. 100, (ed. E. Athanassoula, Dordrecht: Reidel), 391.

Frenk, C.S., 1986. Phil. Trans. R. Soc., 330, 517.

Frenk, C.S., 1987. In Nearly Normal Galaxies: from the Planck Time to the Present, (ed S.M. Faber, Springer-Verlag), 421.

Frenk, C.S., White, S.D.M., Efstathiou, G. and Davis, M., 1985. Nature,317, 595.

Frenk, C.S., White, S.D.M., Davis, M. and Efstathiou, G., 1987. Astrophys. J.,in press.

Haynes, M.P. and Giovanelli, R. 1986. Astrophys. J. Lett., 306, L55.

Kaiser, N. 1987. Preprint.

Kirshner, R.P., Oemler, A., Schechter, P.L. and Shectman, S.A. 1987. Astrophys. J., 314, 493.

de Lapparent, V., Geller, M.J. and Huchra, J.P., 1986. Astrophys. J. Lett., 302, L1.

McGlynn, T.A., 1984. Astrophys. J., 281, 13. 
Negroponte, J. and White, S.D.M., 1983. Mon. Not. R. astr. Soc., 205, 1009.

Nolthenius, R. and White, S.D.M. 1986. Mon. Not. R. astr. Soc., 225, 505.

Peebles, P.J.E., 1982. Astrophys. J. Lett.,263, L1.

Quinn, P.J., Salmon, J.K. and Zurek, W.H., 1986. Nature, 322, 392.

Rubin, V.C., Burstein, D., Ford, W.K. and Thonnard,N., 1985. Astrophys. J., $289,81$.

Toomre, A., 1977. In Evolution of Galaxies and Stellar Populations, (ed B.M. Tinsley and R.B. Larson, Yale Univ. Obs.), 401.

Tully, R.B. and Fisher, J.R., 1977. Astron. Astrophys., 54, 661.

Vittorio, N., Juszkiewicz,R. and Davis, M. 1986. Nature, 323, 132.

Vittorio, N. and Silk, J. 1984. Astrophys. J., 285, L39.

White, S.D.M. 1985. Pap. presented at CITA Conf. on Galaxy Formation (Toronto).

White, S.D.M. 1986. In Inner Space/Outer Space, (ed. E.W.Kolb, M.S.Turner, D.Lindley, K.Olive and D.Seckel, University of Chicago Press), 228.

White, S.D.M., Davis, M., Efstathiou, G. and Frenk, C.S. 1987a. Nature, in press. White, S.D.M., Frenk, C.S., Davis, M. and Efstathiou, G., 1987b. Astrophys. J., 313, 505.

White, S.D.M. and Rees, M.J., 1978. Mon. Not. R. astr. Soc., 183, 341. 